\title{
GRAIN CHARGING AND SHIELDING PROCESSES IN COLLISIONAL PLASMAS
}

\author{
S.YA. BRONIN, L.G. D'YACHKOV, A.G. KHRAPAK, S.V. VLADIMIROV
}

PACS 52.27.Lw

(C) 2011
Joint Institute for High Temperatures, Russian Academy of Sciences

(13, Izhorskaya Str., Moscow 125412, Russia; e-mail: khrapak@mail.ru)
Effects of the volume processes of plasma ionization and recombination are usually disregarding for the simplification of analytical models of charging and shielding of dust grains in complex plasmas. However, these effects can be important in many cases, and they should be taken into account in self-consistent theories of dust grain charging and shielding in bulk structures. In this paper, we generalize our theoretical model for highly collisional plasma (D'yachkov L.G. et al., Phys. Plasmas 14, 042102 (2007)) with regard for the volume ionization and recombination.

\section{Introduction}

The problem of plasma shielding and charging of macroscopic (with respect to the sizes of electrons and ions) objects such as dust grains is one of the most important ones in complex plasmas [1-3]. There are many plasma processes which influence the shielding and charging, including the effects of absorption and emitting of plasma particles by a grain, of plasma particle collisions, and volume processes of plasma ionization and recombination. These effects are important for the interaction of dust grains in dust clouds, clusters, and crystals [4] and for related phenomena such as phase transitions in complex plasma structures $[5,6]$. It was recently shown [7] that the dust shielding by plasma is changed qualitatively if the ion absorption by a grain is taken into account: instead of a Debye-like potential at large distances, the $r^{-2}$ tail appears in the potential in the case of collisionless plasmas. Here, we consider the case of highly collisional plasma where the electron and ion mean free paths are smaller than other characteristic lengths of the problem, except possibly the grain radius. A similar case without accounting the volume ionization and recombination processes was considered in [8]. Now, we take these processes into account.

\section{Theoretical Model}

The stationary distributions of the electric field as well as the electron and ion concentrations around a dust particle, whose surface absorbs and emits charged components of plasma, are considered. The analytic solution of this problem with regard for the volume processes of plasma ionization and recombination was obtained. The continuity equation for plasma ions (electrons) is given by

$\frac{\partial n_{i(e)}}{\partial t}+\nabla \mathbf{j}_{i(e)}=S_{i}-S_{r}$

where $\mathbf{j}_{i(e)}$ is the ion (electron) flux density, $S_{i}$ and $S_{r}$ are the ionization and recombination rates, respectively. If the ion $\ell_{i}$ and electron $\ell_{e}$ mean free paths are small with respect to other characteristic lengths of the problem such as the grain radius $a$ and the plasma screening length $\lambda$, the drift-diffusion (continuum) approximation is applicable. We thus obtain

$\mathbf{j}_{i(e)}=\mp n_{i(e)} \mu_{i(e)} \nabla \varphi-D_{i(e)} \nabla n_{i(e)}$,

where $\varphi$ is the electrostatic potential, $\mu_{i(e)}$ and $D_{i(e)}$ are, respectively, the mobility and diffusion coefficients related by the Einstein's formula $\mu_{i(e)} T_{i(e)}=e D_{i(e)}$. For the spherically symmetric problem, we have in the stationary state [9]

$\frac{d n_{i(e)}}{d r} \pm \frac{e n_{i(e)}}{T_{i(e)}} \frac{\mathrm{d} \varphi}{\mathrm{d} r}+\frac{I_{i(e)}}{4 \pi D_{i(e)} r^{2}}=0$

where $I_{i(e)}=4 \pi r^{2} j_{i(e)}$ is the total ion (electron) flux from the grain. In the stationary state, the ion and electron fluxes are equal $\left(I_{i}=I_{e}=I\right)$ and determined by

$\frac{d I}{d r}=4 \pi r^{2} S(r)$

where $S=S_{i}-S_{r}$. Equations (3) and (4) together with the Poisson's equation

$\frac{1}{r^{2}} \frac{d}{d r}\left(r^{2} \frac{d \varphi}{d r}\right)=-4 \pi e\left(n_{i}-n_{e}\right)$

form a set of four equations for four unknown functions $n_{i}, n_{e}, I$, and $\varphi$. 
We assume that the volume ionization and recombination processes are insignificant within the region of radius $\sim \lambda$, where the charge separation is large. Outside of this region, the charge separation is small, and $n_{i}=n_{e}=n$. Let $n_{0}$ be the equilibrium plasma concentration in the absence of dust grains. When considering $S$ as a function of $n$, we restrict ourselves to the linear approximation in the expansion of $S$ in powers of $\left(n_{0}-n\right)$

$$
S \cong \frac{n_{0}-n}{\tau}, \quad \tau^{-1}=-\left.\frac{d S}{d n}\right|_{n_{0}}
$$

The inequality $\tau>0$ represents the condition of plasma stability. Close to the dust grain, gradients of the potential $\varphi$ as well as the ion $n_{i}$ and electron $n_{e}$ densities are large enough, and the volume ionization and recombination processes can be neglected. To estimate the radius of this region, we apply the operator $\frac{1}{r^{2}} \frac{d}{d r} r^{2}$ to Eqs. (3):

$$
\frac{1}{r^{2}} \frac{d}{d r}\left(r^{2} \frac{d n_{i(e)}}{d r}\right) \pm \frac{e}{T_{i(e)}} \frac{1}{r^{2}} \frac{d}{d r}\left(r^{2} n_{i(e)} \frac{d \varphi}{d r}\right)=-\frac{S(r)}{D_{i(e)}}
$$

Taking into account that $n_{0}$ is the natural scale for $n_{i(e)}$, one can estimate the first term on the left-hand side of Eq. (7) as $n_{0} / r^{2}$ and the right-hand side of Eq. (7) as $n_{0} / D_{i(e)} \tau$. It follows from the comparison of these estimates that the volume ionization and recombination processes can be neglected when $r \ll \sqrt{D_{i} \tau}$ (we take into account that $\left.D_{i} \ll D_{e}\right)$. Thus, the condition of insignificance of the ionization and recombination processes in the region where the charge separation is significant can be written as the inequality $\lambda \ll \sqrt{D_{i} \tau}$. We also suggest that $\lambda \ll L$, where $L=\left(3 / 4 \pi n_{d}\right)^{1 / 3}$ is the Wigner-Seitz radius, and $n_{d}$ is the dust grain number density.

The outward boundary conditions at $r=L$ require the equality to zero of the spatial derivatives of all values, as well as the absence of a charge flow $(I(L)=0)$. The inner boundary conditions should be specified at the boundary of the collisionless layer $\left(r=a+\ell_{i(e)}\right.$, where $\ell_{i(e)}$ are the ion and electron mean free paths) in the form of a relation between the concentrations and the fluxes of plasma particles, which follow from the consideration of the motion of particles inside this layer in the electric field. The electric field in the collisionless layer is considered equal to $e^{2} Z_{d} / r$, where $Z_{d}$ is the particle charge, which itself is determined from solution of Eqs. (3)-(5). It is assumed that charged particles recombine at the dust particle surface. The simple analytical solution of the aforesaid problem is possible when the inequalities $a \ll \ell_{i(e)} \ll L$ are fulfilled. The implementation of this inequality allows dividing the integration interval into three regions:

(I) $r \sim a+\ell_{i(e)} \ll \lambda$,

(II) $r \sim \lambda$,

(III) $\lambda \ll r<L$.

Such a division allows us to simplify the problem in each region; the general solution appears by matching the corresponding partial solutions at the boundaries between them.

The dust particle charge obtained from the general solution of the problem is given by

$\frac{e^{2} Z_{d}}{a T_{e}}=\ln \frac{D_{e}}{D_{i}}+\ln G$

In two limit cases, we have

$G=\frac{T_{i}}{T_{e}} \frac{1-\exp \left(-r_{i} / a\right)}{1-\exp \left(-r_{e} / a\right)}, \quad \ell_{i(e)} \ll a$,

$G=\frac{\ell_{i}}{\ell_{e}} \frac{1}{1+r_{i} / a}, \quad \ell_{i(e)} \gg a$.

Here, the values of $r_{i(e)}$ depend on $Z_{d}\left(r_{i(e)}=\right.$ $\left.e^{2} Z_{d} / T_{i(e)}\right)$, thus (8) is in fact the equation for the determination of $Z_{d}$, which is easy solved by simple iterations.

The plasma density in region (III), i.e., in all space with the exception of thin screening layers adjacent to particle surfaces, is given by

$n \cong n_{0}\left[1+\frac{1}{4 \pi D \beta R} \frac{\cosh x-x \sinh x}{x \cosh x-\sinh x}\right]^{-1}$.

Here,

$\beta=\left[\sqrt{2 \pi} \frac{\left(1-\gamma_{e}\right) r_{e} \ell_{e}}{a^{2}} e^{r_{e} / a}+e^{r_{e} /\left(a+\ell_{e}\right)}-1\right] \frac{1}{4 \pi D_{e} r_{e}}$,

where $\gamma_{e}$ is a coefficient depending on the parameter $\ell_{e} / a$ and characterizing the influence of the electron absorption on the plasma density at the outward boundary of the collisionless region $\left(0<\gamma_{e}<1 / 2\right), x=L / R$, $R=\sqrt{D \tau}$, and $D=\left(T_{i}+T_{e}\right) /\left(T_{i} / D_{i}+T_{e} / D_{e}\right)$.

The flux of electrons and ions $I_{0}=I(a)$ at the dust particle surface is given by

$I_{0}=-\frac{n_{0}}{\beta}\left[1+\frac{1}{4 \pi D \beta R} \frac{\cosh x-x \sinh x}{x \cosh x-\sinh x}\right]^{-1}=-\frac{n}{\beta}$. 
The minus sign corresponds to the fact that the flux is directed to the particle surface. The value of $I_{0}$ determines a role of the surface absorption of charged plasma particles in their ionization/recombination balance.

For $x$ being not too small (not too large number density of dust grains $n_{d}=3 / 4 \pi x^{3} R^{3}$ ), the second term in the brackets in Eq. (9) can de neglected; this results in $n \cong n_{0}$. This term becomes of the order of 1 for small $x$, when the approximations $x \cosh x-\sinh x \cong x^{3} / 3$ and $\cosh x-x \sinh x \cong 1$ are valid. In this case, we have

$n \cong n_{L}=\frac{n_{0} \alpha}{1+\alpha}, \quad I_{0}=-\frac{n_{0}}{\beta} \frac{\alpha}{1+\alpha}$,

where

$\alpha=\frac{4 \pi L^{3}}{3 \tau} \beta$

The physical meaning of the parameter $\alpha$ becomes clear if we rewrite it in the following form:

$\alpha=\frac{4 \pi L^{3}}{3} \frac{n}{\tau} / \frac{n}{\beta}$

Thus, $\alpha$ is equal to the ratio of the ionization rate in the Wigner-Seitz cell to the recombination rate on the grain surface at the maximum value of the flux $I_{0}=-n_{0} / \beta$. So, the parameter $\alpha$ characterizes the relation between the volume and surface recombinations: for $\alpha \ll 1$, the plasma density is determined by the balance of the volume ionization and the surface recombination, while, at $\alpha \gg 1$, the surface recombination does not affect the plasma density. In the above-considered case of not so small $x$, the surface recombination does not play any role.

\section{Conclusions}

We have derived a theory of dust grain charging and shielding in highly collisional plasma in the case of the electron and ion mean free paths and the grain radius smaller than the plasma screening length with an arbitrary relation between the mean free paths and the grain radius. The effects of the volume processes of plasma ionization and recombination are also considered.
This work was partly supported by the Russian Foundation for Basic Research (project No. 08-02-00444) and jointly by the Joint Institute for High Temperatures and Max Planck Institut für Extraterrestrische Physik (Partner Group for Complex Plasma).

1. V.E. Fortov, A.G. Khrapak, S.A. Khrapak, V.I. Molotkov, and O.F. Petrov, Physics-Uspekhi 47, 447 (2004).

2. S.V. Vladimirov and K. Ostrikov, Phys. Rep. 393, 175 (2004).

3. V.E. Fortov, A.V. Ivlev, S.A. Khrapak, A.G. Khrapak, and G.E. Morfill, Phys. Rep. 421, (2005).

4. V.N. Tsytovich, G.E. Morfill, S.V. Vladimirov, and H. Thomas, Elementary Physics of Complex Plasmas (Springer, Berlin, 2007).

5. S.A. Khrapak, G.E. Morfill, A.V. Ivlev, H.M. Thomas, D.A. Beysens, B. Zappoli, V.E. Fortov, A.M. Lipaev, and V.I. Molotkov, Phys. Rev. Lett. 96, 015001 (2006).

6. S.A. Khrapak and G.E. Morfill, Phys. Rev. Lett. 103, 255003 (2009).

7. S.A. Khrapak, B.A. Klumov, and G.E. Morfill, Phys. Rev. Lett. 100, 225003 (2008).

8. L.G. D'yachkov, A.G.Khrapak, S.A. Khrapak, and G.E. Morfill, Phys. Plasmas 14, 042102 (2007).

9. C.H. Su and S.H. Lam, Phys. Fluids 6, 1479 (1963).

Received 17.03.11

ПРОЦЕСИ ЗАРЯДКИ ТА ЕКРАНУВАННЯ ПИЛОВИХ ЧАСТИНОК У ЗІТКНЕНИХ ПЛАЗМАХ

С.Я. Бронін, Л.Г. Дячков, А.Г. Храпак, С.В. Владимиров

$\mathrm{P}$ е $з$ ю м е

Ефекти об'ємних процесів іонізації і рекомбінації в плазмі зазвичай не розглядаються для спрощення аналітичних моделей зарядки та екранування пилових частинок в комплексній плазмі. Але, ці ефекти можуть бути важливими в багатьох випадках і їх потрібно враховувати в самоузгоджених теоріях зарядки та екранування пилових частинок в об'ємних структурах. У даній роботі узагальнено нашу теоретичну модель сильно зіткненої плазми (Л.Г. Дьячков и др., Phys. Plasmas 14, 042102 $(2007))$, враховуючи іонізацію і рекомбінацію в об'ємі. 